\title{
Circulating Angiogenic Growth Factors in Diabetes Patients with Peripheral Arterial Disease and Exertional Leg Pain in Ghana
}

\author{
Kwame Yeboah, ${ }^{1}$ Jennifer A. Agyekum, ${ }^{2}$ Eric Kyei Baafour, ${ }^{3}$ Daniel A. Antwi, \\ Afua B. Adjei, ${ }^{4}$ Vincent Boima, ${ }^{5}$ and Ben Gyan ${ }^{3}$ \\ ${ }^{1}$ Department of Physiology, School of Biomedical \& Allied Health Sciences, University of Ghana, Accra, Ghana \\ ${ }^{2}$ Department of Medical Laboratory Sciences, School of Biomedical \& Allied Health Sciences, University of Ghana, Accra, Ghana \\ ${ }^{3}$ Department of Immunology, Noguchi Memorial Institute for Medical Research, University of Ghana, Accra, Ghana \\ ${ }^{4}$ Department of Chemical Pathology, School of Biomedical \& Allied Health Sciences, University of Ghana, Accra, Ghana \\ ${ }^{5}$ Department of Medicine \& Therapeutics, School of Medicine \& Dentistry, University of Ghana, Accra, Ghana
}

Correspondence should be addressed to Kwame Yeboah; melvinky@gmail.com

Received 23 August 2017; Revised 27 November 2017; Accepted 29 November 2017; Published 27 December 2017

Academic Editor: Robert M. Schainfeld

Copyright (C) 2017 Kwame Yeboah et al. This is an open access article distributed under the Creative Commons Attribution License, which permits unrestricted use, distribution, and reproduction in any medium, provided the original work is properly cited.

\begin{abstract}
Objective. Peripheral arterial disease (PAD) is a common complication of diabetes, associated with impairment in angiogenesis. Angiogenesis is regulated by angiogenic growth factors such as angiopoietin 1 (Ang-1), Ang-2, and vascular endothelial growth factor (VEGF). We studied the association between angiogenic growth factors versus PAD and exertional leg symptoms in diabetes patients in Ghana. Method. In this cross-sectional study, ankle-brachial index was measured with oscillometrically and exertional leg symptoms were screened with Edinburgh claudication questionnaire in 140 diabetes patients and 110 nondiabetes individuals. Circulating levels of Ang-1, Ang-2, and VEGF were measured with immunosorbent assay. Results. The prevalence of PAD and exertional leg pain was $16.8 \%$ and $24.8 \%$, respectively. Compared to non-PAD participants, PAD patients had higher VEGF levels [85.8 (37.5-154.5) versus 57.7 (16.6-161.1) $p=0.032$ ] and lower Ang-1 levels [31.3 (24.8-42.6) versus 40.9 (28.2-62.1), $p=0.017]$. In multivariable logistic regression, patients with exertional leg pain had increased the odds of plasma Ang-2 levels [OR (95\% CI): 2.08 (1.08-6.41), $p=0.036$ ]. Conclusion. Diabetes patients with PAD and exertional leg pain have imbalance in angiogenic growth factors, indicating impaired angiogenesis. In patients with exertional leg pains, Ang-2 may be an important biomarker.
\end{abstract}

\section{Introduction}

Peripheral arterial disease (PAD) is the formation of atherosclerotic plaque in non-coronary vessel and preferentially affects the lower extremities. PAD is the third leading cause of atherosclerotic vascular death after coronary heart disease and stroke. Globally, it was estimated in 2013 that PAD affects more than 202 million individuals [1]. The prevalence of PAD in sub-Sahara African population is largely undetermined. Our previous studies indicated that PAD is common among non-smoking Ghanaians, especially in diabetes patients [2]. Arterial obstruction in PAD may be presented as leg pain during exertional activities, attributed to inadequate skeletal muscle perfusion to meet the increased metabolic demands [3]. The Edinburgh claudication questionnaire (ECQ) can be used to screen for symptoms associated with such symptomatic PAD [4]. However, more than $70 \%$ of PAD patients are asymptomatic and clinical signs may appear at late stages of the disease [5]. In such patients, ankle-brachial index (ABI), which is a simple, highly reproducible, noninvasive hemodynamic test, can be used to screen for PAD. ABI $<0.9$ may also imply the presence of systemic atherosclerosis [6].

Angiogenic growth factors are endogenous molecules that regulate growth and development of the microvessels [7]. Most studies have reported vascular endothelial growth factor (VEGF), angiopoietin 1 (Ang-1), and angiopoietin 2 (Ang-2) to be potent regulators of microvascular remodelling in health and atherosclerotic diseases [8]. Ang-1 is expressed in non-endothelial cells, such as pericytes, smooth muscle 
cells, and fibroblast, whereas the major source of Ang-2 is from the endothelial cells [7]. In PAD patients, atherosclerotic occlusion results in ischemia of tissues distal to the plaque. This stimulates varying degrees of collateral blood vessel formation mediated by circulating angiogenic growth factors. Ang-2/VEGF promotes vessel sprouting and proliferation, whereas Ang-1 promotes circumferential growth required for vessel maturation and stabilization [8]. The degree of functional collateral blood vessel formation may alter the clinical manifestations of PAD. We, therefore, studied the levels of circulating angiogenic growth factors, Ang-1, Ang2 , and VEGF, in diabetes patients with PAD and leg pain on exertion. We hypothesize that, compared to individuals without PAD, PAD patients have imbalance in angiogenic growth factors.

\section{Methods}

2.1. Design and Setting. This study was conducted within the period of December 2012 to June 2013, at the Korle-Bu Teaching Hospital in Accra, which is a 1500-bed tertiary hospital and serves as the main referral hospital in Ghana. ABI was measured in all the study population, which were selected from two sources: (1) diabetes patients, selected systematically as every 3 rd consecutive patient visiting the diabetes clinic and consented to take part in the study, and (2) nondiabetic individuals, invited from the surrounding communities and conveniently recruited into the study. All the study participants were categorised as PAD (ABI < 0.9 ) and non-PAD (ABI: 0.9-1.3) based on the ABI values. Individuals with stiff/incompressible arteries (ABI > 1.3), history/medication of CVDs, and those unable to comprehend and comply with the protocol requirements (psychological and/or cognitive disorders, failure to cooperate, and failure to sign the informed consent document) were excluded from the study. In all, 250 subjects, comprising 140 diabetes patients and 110 nondiabetic individuals, were screened for PAD. This study was conducted according to the tenets of the Declaration of Helsinki of 1975 (1983 revision) and was carried out with the approval of the University of Ghana Medical School Ethical and Protocol Review Committee (protocol ID number: MS-Et/M.2-P.4.10/2012-2013). All the study participants gave written informed consent after the procedures involved in the study were thoroughly explained to them.

2.2. Anthropometry and BP Measurement. Weight, height, waist, and hip circumferences were measured using standard protocol [9]. Briefly, body weight was measured twice using a homologated electronic scale (Seca 770) following due calibration (precision $\pm 0.1 \mathrm{~kg}$ ), with the patient wearing light clothing with shoes removed. Height was also measured with a portable system (Seca 222) with the patient shoeless in the upright position. Body mass index (BMI) was calculated as weight $(\mathrm{kg})$ divided by height squared $\left(\mathrm{m}^{2}\right)$. Waist circumference was measured with nonelastic tape measure at the upper border of the iliac crest, parallel to the floor without compressing the skin. Blood pressure was measured three times, with a validated Blood Pressure
Monitor (Omron 991X, Omron Health Care, Japan), at the right upper arm of participants with an appropriate cuff size, after at least 5 min rest, seated comfortably with arm and back support. Hypertension was defined as subjects with BP $\geq 140 / 90 \mathrm{mmHg}$ and/or on antihypertensive medication.

2.3. ABI and Exertional Leg Pain Assessment. Ankle and brachial blood pressures were measured in all participants after a minimum of $5 \mathrm{~min}$ rest in a supine position on an examination table in a temperature controlled room using automated oscillometric method (Vasera 1500N, FukudaDenshi, Tokyo). The BP cuffs of the Vasera were applied to both arms and ankles to simultaneously measure BPs. ABI was calculated for each leg as the ratio systolic BP in the ankle divided by the higher of systolic blood pressure in the arm. ABI $>0.9$ was considered normal and PAD was defined as $\mathrm{ABI} \leq 0.9$ in at least one leg.

Leg symptoms of patients were assessed using the ECQ and categorised based on previous work by Hirsch et al. [10]. The presence of exertional leg pain was defined as patients with classical intermittent claudication (exertional calf symptoms that do not begin at rest worsen when walking uphill or hurrying and resolve within $10 \mathrm{~min}$ of rest), atypical intermittent claudication (exertional calf symptoms that do not begin at rest but are otherwise not consistent with classical intermittent claudication), as well as rest pains (exertional leg symptoms that also begin at rest).

2.4. Biochemical Analysis. Blood samples were drawn in the morning, after 8-12 hours of overnight fasting. Fasting plasma glucose (FPG), 2-hour postglucose load plasma glucose (2 h PPG), total cholesterol, high-density lipoprotein cholesterol (HDL), triglyceride, and creatinine were analysed using BS 400 chemical autoanalyser (Mindray, China) and commercial reagents (Randox Laboratory Reagents, UK). Low-density lipoprotein (LDL) cholesterol levels were calculated using Friedewald's formula.

Serum levels of Ang-1, Ang-2, and VEGF were measured by sandwich enzyme-linked immunosorbent assay, using commercially available enzyme-linked immunosorbent assay kits (R\&D Systems, Minneapolis, MN). The assays were performed according to the manufacturer's recommendations and the total interassay coefficient of variation for the three assays were $<7 \%$. Lowest limit of the detection were $0.03 \mathrm{ng} / \mathrm{ml}$ for VEGF, $0.16 \mathrm{ng} / \mathrm{ml}$ for Ang-1, and $0.06 \mathrm{ng} / \mathrm{ml}$ for Ang-2.

2.5. Statistical Analysis. Continuous data were analysed with the Shapiro-Wilk test to determine their distribution. Variables with normal distribution were presented as mean \pm standard deviation and analysed using Student's $t$-test. Variables with nonnormal distribution were presented as median and interquartile range and analysed using Mann-Whitney $U$ test. Categorical data were analysed by the $\chi^{2}$ test. Multivariable logistic regression models were performed to compute adjusted and unadjusted odd ratios between (1) angiogenic growth factors versus PAD (low ABI) and (2) angiogenic growth factors versus leg pain on exertion. 
TABLE 1: General characteristics of study participants by diabetes status.

\begin{tabular}{|c|c|c|c|c|}
\hline & All participants $(n=250)$ & $\mathrm{T} 2 \mathrm{DM}(n=140)$ & Non-DM $(n=110)$ & $p$ \\
\hline Females, $n(\%)$ & $125(50)$ & $63(45)$ & $62(56.4)$ & 0.15 \\
\hline Age, yrs & $54.1 \pm 10.2$ & $53.7 \pm 10.1$ & $54.6 \pm 10.3$ & 0.54 \\
\hline Weight, kg & $79.5 \pm 14.9$ & $79.9 \pm 15.5$ & $79 \pm 14.3$ & 0.672 \\
\hline Height, cm & $166 \pm 8.4$ & $167 \pm 8$ & $164 \pm 9$ & 0.061 \\
\hline $\mathrm{BMI}, \mathrm{kg} / \mathrm{m}^{2}$ & $29.1 \pm 5.7$ & $28.9 \pm 5.9$ & $29.4 \pm 5.5$ & 0.571 \\
\hline Waist circumference, $\mathrm{cm}$ & $98 \pm 14$ & $99 \pm 12$ & $96 \pm 15$ & 0.073 \\
\hline Waist-hip ratio & $0.91 \pm 0.11$ & $0.92 \pm 0.07$ & $0.9 \pm 0.14$ & 0.382 \\
\hline Systolic BP, mmHg & $139 \pm 30$ & $141 \pm 26$ & $135 \pm 34$ & 0.174 \\
\hline Diastolic BP, mmHg & $83 \pm 13$ & $83 \pm 13$ & $82 \pm 14$ & 0.594 \\
\hline Pulse BP, mmHg & $59 \pm 14$ & $59 \pm 14$ & $58 \pm 13$ & 0.485 \\
\hline Heart rate, bpm & $71 \pm 17$ & $75 \pm 13$ & $65 \pm 19$ & $<0.01$ \\
\hline $\mathrm{FPG}, \mathrm{mmol} / \mathrm{l}$ & $6.9 \pm 3.2$ & $8.4 \pm 2.9$ & $5 \pm 2.5$ & $<0.01$ \\
\hline $2 \mathrm{~h}$ PPG, mmol/l & $7.8 \pm 1.4$ & & $7.8 \pm 1.4$ & \\
\hline Triglycerides, mmol/l & $1.1 \pm 0.5$ & $1.1 \pm 0.5$ & $1.2 \pm 0.6$ & 0.586 \\
\hline Total cholesterol, mmol/l & $4.7 \pm 1.5$ & $5.5 \pm 1.4$ & $3.9 \pm 1.1$ & $<0.001$ \\
\hline HDL cholesterol, mmol/l & $0.9 \pm 0.2$ & $0.7 \pm 0.2$ & $1.2 \pm 0.4$ & 0.025 \\
\hline LDL cholesterol, mmol/l & $3.2 \pm 1.4$ & $3.9 \pm 1.3$ & $2.7 \pm 1.4$ & $<0.001$ \\
\hline \multicolumn{5}{|l|}{ Serum creatinine mg/dl } \\
\hline $\mathrm{eGFR} \mathrm{ml} / \mathrm{min} / 1.73 \mathrm{~m}^{2}$ & $82.4(73.1-104.9)$ & $70.8(51.4-82.1)$ & $105.9(76.2-118.7)$ & $<0.001$ \\
\hline Low eGFR, $n(\%)$ & $43(17.2)$ & $34(24.3)$ & $9(8.2)$ & 0.001 \\
\hline Angiopoietin 1, ng/ml & $38.2(25.7-47.9)$ & $36.1(24.7-42.1)$ & $41.1(30-57.3)$ & 0.01 \\
\hline Angiopoietin 2, pg/ml & $740(392-1107)$ & $838(473-1241)$ & $597(274-1005)$ & 0.018 \\
\hline VEGF-A, pg/ml & $63.2(21.2-157.8)$ & $72.2(28-201.8)$ & $48.4(17.4-110.1)$ & 0.025 \\
\hline VPT, mV & $11.9 \pm 6.2$ & $15.1 \pm 7.8$ & $7.3 \pm 3.8$ & $<0.001$ \\
\hline Right ABI & $0.98 \pm 0.18$ & $0.92 \pm 0.21$ & $1.17 \pm 0.13$ & 0.135 \\
\hline Left ABI & $0.97 \pm 0.13$ & $0.91 \pm 0.12$ & $1.15 \pm 0.14$ & 0.132 \\
\hline Right leg PAD & $36(14.4)$ & $29(20.7)$ & $7(6.4)$ & $<0.001$ \\
\hline Left leg PAD & $26(10.4)$ & $21(15)$ & $6(5.5)$ & $<0.001$ \\
\hline Leg pains on exertion & $62(24.8)$ & $43(37.7)$ & $19(17.3)$ & 0.004 \\
\hline
\end{tabular}

\section{Results}

Diabetes patients had a higher proportion of hypertensives and had higher heart rate, FPG, total cholesterol, and LDL cholesterol levels, as well as lower HDL cholesterol levels. Compared to nondiabetes participants, diabetes patients had higher prevalence of PAD and low eGFR. For circulating angiogenic growth factors levels, compared to nondiabetes participants, diabetes patients had higher levels of plasma Ang-2 and VEGF and lower levels of Ang-1 (Table 1). The average duration of diabetes was $8.5 \pm 6.9$ years, higher in diabetes patients with PAD $(12.1 \pm 9.5$ versus $6.4 \pm 4.8$ years, $p=$ $0.028)$ or exertional leg pain ( $10.6 \pm 7.1$ versus $6.8 \pm 5.3$ years, $p=0.044)$, compared to patients without PAD or exertional leg pain, respectively. With respect to treatment of diabetes, $18(12.9 \%)$ patients were on diet and lifestyle modification, 56 (40\%) patients were on oral hypoglycaemic medication only and $66(47.1 \%)$ were treated with both oral hypoglycaemics and injectable insulin. Compared to diabetes patients with PAD, patients without PAD (71.4\% versus $10.9 \%$ ) were more likely to be treated with oral hypoglycaemics and insulin [OR $=2.23(1.08-4.59), p=0.03]$.
The overall prevalence of PAD (low $\mathrm{ABI}$ ), in at least one leg, was $16.8 \%$, with $14.4 \%$ of the participants having PAD in the right leg and $10.4 \%$ having PAD in the left leg. The overall prevalence of exertional leg pain was $24.8 \%$ in at least one leg, with $8.8 \%$ of the participants having both PAD and leg pain. Patients with PAD had higher pulse BP, higher proportion of leg pain, diabetes, and hypertension and are more likely to be former smokers and alcohol consumers. Compared to non-PAD participants, PAD patients had higher serum levels of VEGF and lower levels of Ang-1 (Table 2). Compared to participants without leg pain, nondiabetic participants with leg pain had lower levels of serum Ang-1, whereas Ang-1 levels were similar between diabetic participants with or without leg pain (Figure 1). In diabetes and nondiabetes participants, those with leg pain on exertion had higher levels Ang-2 (Figure 2). However, there was no difference in VEGF levels between diabetes and nondiabetes participants with or without leg pain (Figure 3).

In unadjusted logistic regression models, having PAD was significantly associated with odds of increase in VEGF and odds of decrease in Ang-1. However, this association was nonsignificant after adjusting the model for various 
TABLE 2: Clinical characteristics of study participants by PAD status.

\begin{tabular}{|c|c|c|c|c|}
\hline Characteristics & All participants $(n=250)$ & Non-PAD $(n=208)$ & $\operatorname{PAD}(n=42)$ & $p$ \\
\hline Age, years & $54.1 \pm 10.2$ & $53.6 \pm 10.3$ & $58 \pm 8.8$ & 0.062 \\
\hline Female, $n(\%)$ & $127(50.8)$ & $98(47.1)$ & $17(40.5)$ & 0.142 \\
\hline Diabetes, $n(\%)$ & $140(56)$ & $106(51)$ & $34(80.9)$ & 0.002 \\
\hline Hypertension, $n(\%)$ & $152(60.8)$ & $116(55.8)$ & $36(85.7)$ & $<0.001$ \\
\hline Leg pains & $62(24.8)$ & $40(19.2)$ & $22(52.4)$ & $<0.001$ \\
\hline Alcohol intake, $n(\%)$ & $12(4.8)$ & $4(1.9)$ & $8(19)$ & $<0.001$ \\
\hline Previous smokers, $n(\%)$ & $47(18.8)$ & $22(10.6)$ & $25(59.5)$ & $<0.001$ \\
\hline Body height, $\mathrm{cm}$ & $166 \pm 8.4$ & $165 \pm 8$ & $166 \pm 9$ & 0.699 \\
\hline $\mathrm{BMI}, \mathrm{kg} / \mathrm{m}^{2}$ & $29.1 \pm 5.7$ & $29.1 \pm 5.4$ & $29.5 \pm 7.5$ & 0.735 \\
\hline Body fat, $\%$ & $34.6 \pm 12.6$ & $35.1 \pm 12.6$ & $32.2 \pm 12.9$ & 0.318 \\
\hline Visceral fat, $\%$ & $11.2 \pm 4.3$ & $11.2 \pm 4$ & $12.2 \pm 6.2$ & 0.301 \\
\hline Waist circumference, $\mathrm{cm}$ & $98 \pm 14$ & $97 \pm 13$ & $101 \pm 14$ & 0.321 \\
\hline Waist-hip ratio & $0.91 \pm 0.11$ & $0.91 \pm 0.11$ & $0.93 \pm 0.08$ & 0.287 \\
\hline Systolic BP, mm Hg & $139 \pm 30$ & $139 \pm 24$ & $142 \pm 55$ & 0.629 \\
\hline Diastolic BP, mm Hg & $83 \pm 13$ & $82 \pm 13$ & $89 \pm 18$ & 0.124 \\
\hline Pulse BP, mm Hg & $59 \pm 14$ & $58 \pm 13$ & $68 \pm 17$ & 0.027 \\
\hline Mean BP, mm Hg & $102 \pm 16$ & $102 \pm 14$ & $112 \pm 21$ & 0.06 \\
\hline Heart rate, bpm & $71 \pm 17$ & $71 \pm 15$ & $71 \pm 29$ & 0.937 \\
\hline $\mathrm{FPG}, \mathrm{mmol} / \mathrm{L}$ & $6.9 \pm 3.2$ & $6.8 \pm 3.3$ & $7.1 \pm 2.7$ & 0.673 \\
\hline $2 \mathrm{~h} \mathrm{PPG}, \mathrm{mmol} / \mathrm{L}$ & $7.8 \pm 4.1$ & $7.6 \pm 3.8$ & $9.8 \pm 7.1$ & 0.221 \\
\hline Total cholesterol, mmol/L & $4.7 \pm 1.5$ & $4.7 \pm 1.5$ & $4.7 \pm 1.5$ & 0.981 \\
\hline Triglycerides, mmol/L & $1.1 \pm 0.5$ & $1.1 \pm 0.5$ & $1.1 \pm 0.5$ & 0.626 \\
\hline $\mathrm{HDL}, \mathrm{mmol} / \mathrm{L}$ & $0.73 \pm 0.22$ & $0.73 \pm 0.23$ & $0.71 \pm 0.15$ & 0.695 \\
\hline $\mathrm{LDL}, \mathrm{mmol} / \mathrm{L}$ & $3.3 \pm 1.4$ & $3.2 \pm 1.5$ & $3.4 \pm 1.4$ & 0.642 \\
\hline $\mathrm{eGFR} \mathrm{ml} / \mathrm{min} / 1.73 \mathrm{~m}^{2}$ & $82.4(73.1-104.9)$ & $86.3(72.2-106.1)$ & $80.9(71.4-101.8)$ & 0.542 \\
\hline Low eGFR, $n(\%)$ & $43(17.2)$ & $32(15.4)$ & $11(26.2)$ & 0.095 \\
\hline \multicolumn{5}{|l|}{ Vascular growth factors } \\
\hline Ang-1, ng/ml & $38.3(25.7-47.9)$ & $40.9(28.2-62.1)$ & $31.3(24.8-42.6)$ & 0.017 \\
\hline Ang-2,pg/ml & 739.7 (391.7-1106.9) & $732.2(391.7-1098.8)$ & $858.8(416.7-1559.2)$ & 0.174 \\
\hline VEGF-A, pg/ml & $63(21-158)$ & 57.7 (16.6-161.1) & 85.8 (37.5-154.5) & 0.032 \\
\hline Ang-1/2 & $46.3(27.6-90.6)$ & 48.8 (29.7-93) & $38.9(20.9-69.7)$ & 0.081 \\
\hline
\end{tabular}

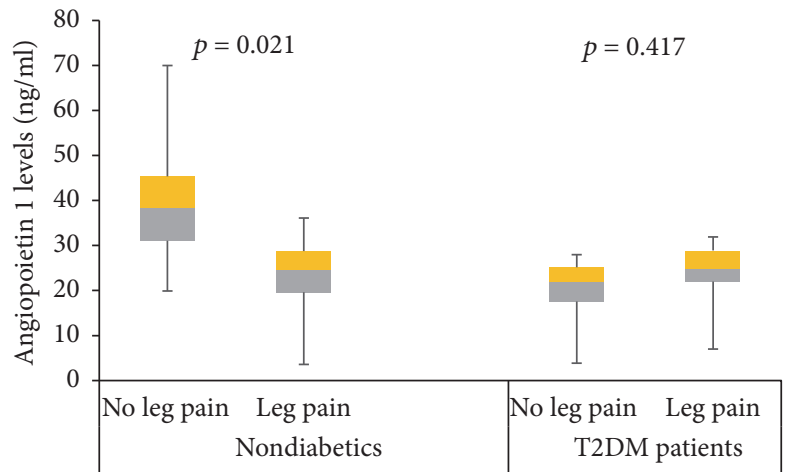

FIgure 1: Circulating levels of angiopoietin 1 among patients with leg pains based on their diabetes status. Data presented as median interquartile range and analysed with Mann-Whitney test.

covariates (Table 3). Patients with leg pain had an increase in odds of serum levels of Ang-1 and Ang-2 in unadjusted

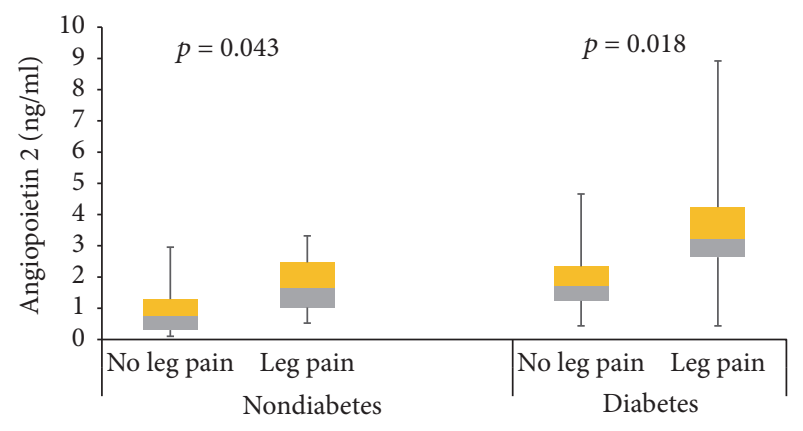

Figure 2: Circulating levels of angiopoietin 2 among patients with leg pains based on their diabetes status. Data presented as median interquartile range and analysed with Mann-Whitney test.

logistic regression model, but only Ang-2 remained significant after adjusting for various covariates (Table 4). 
TABLE 3: Logistic regression models of peripheral arterial disease and vascular growth factors.

\begin{tabular}{lcccc}
\hline & Crude OR (95\% CI) & $p$ & Adjusted OR (95\% CI) $^{*}$ & $p$ \\
\hline Ang-1 & $0.91(0.56-0.99)$ & 0.024 & $0.84(0.42-1.48)$ & 0.096 \\
Ang-2 & $1.56(0.89-3.06)$ & 0.532 & $1.92(0.76-3.52)$ & 0.427 \\
VEGF & $1.29(1.07-1.97)$ & 0.037 & $1.14(0.92-2.13)$ & 0.104 \\
Ang-1/2 & $1.08(0.66-1.84)$ & 0.347 & $1.21(0.51-2.41)$ & 0.625 \\
\hline
\end{tabular}

${ }^{*}$ Adjusted for age, gender, alcohol status, previous cigarette smoking, BMI, waist-hip ratio, diabetes, hypertension, and total cholesterol. All the angiogenic growth factors were logarithmically transformed to improve skewness before analysis. Ang-1: angiopoietin 1; Ang-2: angiopoietin 2; VEGF: vascular-endothelial growth factor; Ang-1/2: angiopoietin 1/angiopoietin 2 ratio.

TABLE 4: Logistic regression models of leg pains and vascular growth factors.

\begin{tabular}{lcccc}
\hline & Crude OR (95\% CI) & $p$ & Adjusted OR (95\% CI) $^{*}$ & $p$ \\
\hline Ang-1 & $1.11(1.02-2.26)$ & 0.048 & $1.09(0.59-2.01)$ & 0.479 \\
Ang-2 & $3.05(1.14-8.94)$ & 0.012 & $2.08(1.08-6.41)$ & 0.036 \\
VEGF & $1.48(0.35-10.25)$ & 0.747 & $0.69(0.41-9.53)$ & 0.78 \\
Ang-1/2 & $1.16(0.27-2.39)$ & 0.239 & $0.8(0.32-1.99)$ & 0.631 \\
\hline
\end{tabular}

${ }^{*}$ Adjusted for age, gender, alcohol status, previous cigarette smoking, BMI, waist-hip ratio, diabetes, hypertension, and total cholesterol. All the angiogenic growth factors were logarithmically transformed to improve skewness before analysis. Ang-1: angiopoietin 1; Ang-2: angiopoietin 2; VEGF: vascular-endothelial growth factor; Ang-1/2: angiopoietin 1/angiopoietin 2 ratio.

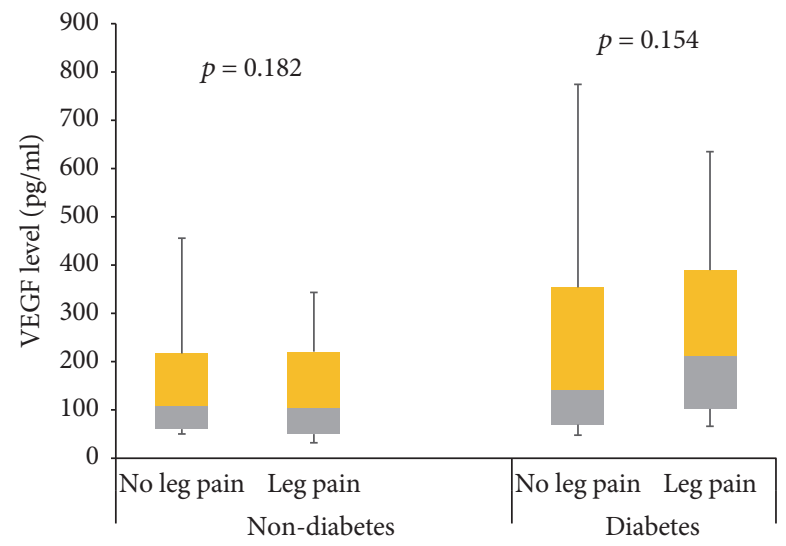

FIGURE 3: Circulating levels of VEGF among patients with leg pains based on their diabetes status. Data represented as median and interquartile range and analysed with Mann-Whitney test.

\section{Discussion}

The major findings of this study are that PAD patients had increased levels of VEGF and decreased levels of Ang-1; however, this was dependent on other CVD risk factors. Also, patients with leg pain on exertion have increased plasma levels of Ang-1 and Ang-2. The association between exertional leg pain and Ang-2 levels was independent of other CVD risk factors in regression model. Studies conducted in the American and British population have shown that patients with PAD have deranged levels of vascular growth factors. Similar to our findings, studies in the UK reported increased plasma VEGF levels in PAD patients [11, 12]. Contrary to the findings of Findley et al. [13] in PAD patients from the United States, we found plasma levels of Ang-2 was similar among $\mathrm{PAD}$ and non-PAD patients in our study.
Peripheral arterial disease is a major health care problem in diabetes patients in Ghana. We reported previously that the prevalence of PAD in diabetes patients in Ghana was 26.7\%, using Doppler measured ABI [14]. In sub-Saharan Africa, ABI is not routinely performed to diagnose PAD, even in high-risk diabetes patients. PAD, therefore, is clinically underrecognized, and management is initiated when associated with symptomatic exertional leg pain. However, most patients with PAD are asymptomatic, and, hence, relying on symptoms before initiating treatment may be less advantageous $[2,14]$. Moreover, the presence of PAD indicates generalised atherosclerosis in such patients. The use of ABI measurement as diagnostic test for PAD is based on the principle that atherosclerotic plaque within the peripheral arterial tree dampens blood flow and pressure towards tissues distal to the plaque, resulting in decreased tissue perfusion and tissue hypoxia. PAD would, therefore, affect capillary modelling and circulating levels of angiogenic growth factors $[15,16]$.

Similar to the findings of this study, imbalance in angiopoietins levels has been reported in diabetes patients elsewhere. Circulating Ang-2 and VEGF levels, but not Ang1 levels, were reported to be associated with endothelial damage in diabetes patients, regardless of the presence of CVD. [17] In hyperlipidaemia patients in Japan [18], compared with nondiabetes patients, diabetes had increased Ang2 levels, while Ang-1 levels were similar between the two patient groups. A population study in Asians indicates that increased levels of Ang-2 were associated with impaired glucose metabolism, diabetes, and hypertension [19]. Likewise, in the Anglo-Scandinavian Cardiac Outcome Studies, compared to nonhypertensives, hypertensive patients had higher levels of plasma Ang-1, Ang-2, and VEGF [20].

Capillary growth or regression is regulated by the balance between proangiogenic factors and antiangiogenic 
factors [8]. Ang-1 regulates vessel maturation and it mediates migration, adhesion, and survival of endothelial cells [21]. Ang-2 is known to cause disruption of connections in endothelial and perivascular cells and promotes cell death and vascular regression. However, in conjunction with VEGF, Ang-2 promotes development of new vessels [8, 21]. Imbalance in vascular growth factors in PAD patients affects angiogenesis in skeletal muscles. Contrary to studies conducted decades ago which reported similar capillarization between PAD patients and controls [22], recent studies have reported reduction in capillary density of muscles of the lower leg of PAD patients [23, 24]. Angiogenic factors, such as Ang1 and VEGF, are required for functional neovascularization in adult tissue in PAD patients [25]. The findings of the current study indicate the imbalance in Ang-1 and VEGF levels in PAD patients, which may affect the ability of the leg muscular tissues to develop new capillaries to adapt to hypoxia induced by atherosclerotic plaque [26].

Compared to participants without leg pain, the levels of Ang-1 decreased, whereas Ang-2 levels increased, in participants with leg pain on exertion. The change was more pronounced in nondiabetes participants. Surprisingly, no change in VEGF level was observed. The reason for this observation may be a result of ethnic differences in vascular growth factors, which has been demonstrated in populations of Caucasians and African Caribbean origins [27]. Also, in our study, we assayed the serum levels of VEGFA using pan-VEGF-A Elisa kits that cannot discriminate between various splicing variants of VEGF-A. Circulating levels of VEGF-A isoforms, arising from differential splicing of exon 8, have important consequence on angiogenesis; VEGF-A $\mathrm{XXX}_{\mathrm{X}}$ isoforms are proangiogenic, while the VEGF$\mathrm{A}_{\mathrm{XXXb}}$ isoforms are antiangiogenic [15]. It has been reported that PAD patients have increased levels of antiangiogenic VEGF- $A_{165 b}$ splicing isoform, and corresponding reduced levels of VEGF- $A_{165 a}$ proangiogenic splicing isoform [16]. Leg pain on exertion reflects inadequate augmentation of skeletal muscle perfusion during exercise, an advanced form of PAD $[13,26]$. Insufficient blood supply produced by arterial ischemia induces a complex program of vascular growth factors dysfunction, resulting in inadequate angiogenesis and collateral vessel formation [26]. Other studies have shown that imbalance in vascular growth factors can be corrected by pharmacological and nonpharmacological interventions $[22,24]$.

\section{Limitations and Conclusion}

The current guideline for PAD diagnosis recommends the use of continuous wave Doppler to measure ABI. In our study, however, ABI was measured using oscillometric method. The oscillometric method is convenient and operator-independent and measures all the limb pressures concurrently. Some studies have reported good concordance between Doppler and oscillometric methods of assessing ABI [28]. Others have reported that, compared to Doppler $\mathrm{ABI}$, oscillometric ABI overestimates the prevalence of PAD. The use of oscillometric method of ABI assessment might be an important limitation in our study [29]. In addition, we did not measure the levels of various splicing variants of VEGF-A isoforms in our study, making the interpretation of circulating levels of VEGF-A difficult. Also, we cannot infer causality from the cross-sectional design of the study. Another important limitation is the inclusion of large numbers of diabetes patients in order to obtain enough PAD patients for analysis since diabetes affects angiogenic growth factors independent of PAD. All the same, this study has shown that PAD patients in sub-Saharan Africa have impaired vascular growth factors levels, an indication of impaired angiogenesis.

\section{Abbreviations \\ PAD: Peripheral arterial disease \\ ABI: Ankle-brachial index \\ ECQ: Edinburgh claudication questionnaire \\ Ang: Angiopoietins \\ VEGF: Vascular endothelial growth factor.}

\section{Additional Points}

Availability of Data and Materials. The dataset supporting the conclusion of this study is available for systematic review and meta-analysis upon request.

\section{Ethical Approval}

The study was conducted in conformity with the Helsinki Declaration on Human Experimentation, 1964, with subsequent revisions, latest Seoul, October 2008. Ethical approval for the study was granted by the Ethics and Protocol Review Committee of the College of Health Science, University of Ghana (Protocol ID no. MS-Et/M.2-P.4.10/2012-2013).

\section{Consent}

All the participants provided voluntary written consent before being recruited into the study.

\section{Conflicts of Interest}

The authors declare that they have no conflicts of interest or financial interest.

\section{Authors' Contributions}

Kwame Yeboah contributed to conception, design, data collection and analysis, and drafting the manuscript and bears the primary responsibility for the content of the manuscript. Daniel A. Antwi, Jennifer A. Agyekum, Afua B. Adjei, and Vincent Boima were involved in the revision of the manuscript. Ben Gyan and Eric Kyei Baafour were involved in laboratory analysis and revision of the manuscript. All the authors read and approved the content of the manuscript.

\section{References}

[1] F. G. R. Fowkes, D. Rudan, I. Rudan et al., "Comparison of global estimates of prevalence and risk factors for peripheral artery disease in 2000 and 2010: a systematic review and analysis," The Lancet, vol. 382, no. 9901, pp. 1329-1440, 2013. 
[2] K. Yeboah, P. Puplampu, E. Yorke, D. A. Antwi, B. Gyan, and A. G. B. Amoah, "Body composition and ankle-brachial index in Ghanaians with asymptomatic peripheral arterial disease in a tertiary hospital," BMC Obesity, vol. 3, no. 1, pp. 1-7, 2016.

[3] N. A. Khan, S. A. Rahim, S. S. Anand, D. L. Simel, and A. Panju, "Does the clinical examination predict lower extremity peripheral arterial disease?" Journal of the American Medical Association, vol. 295, no. 5, pp. 536-546, 2006.

[4] G. C. Lend and F. G. R. Fowkes, "The Edinburgh claudication questionnaire: an improved version of the WHO/Rose Questionnaire for use in epidemiological surveys," Journal of Clinical Epidemiology, vol. 45, no. 10, pp. 1101-1109, 1992.

[5] L. Potier, C. Abi Khalil, K. Mohammedi, and R. Roussel, "Use and utility of Ankle brachial index in patients with diabetes," European Journal of Vascular and Endovascular Surgery, vol. 41, no. 1, pp. 110-116, 2011.

[6] A. Banerjee, F. G. Fowkes, and P. M. Rothwell, "Associations between peripheral artery disease and ischemic stroke: implications for primary and secondary prevention," Stroke, vol. 41, no. 9, pp. 2102-2107, 2010.

[7] G. Y. Koh, "Orchestral actions of angiopoietin-1 in vascular regeneration," Trends in Molecular Medicine, vol. 19, no. 1, pp. 3139, 2013.

[8] E. Fagiani and G. Christofori, "Angiopoietins in angiogenesis," Cancer Letters, vol. 328, no. 1, pp. 18-26, 2013.

[9] World Health Organization, "Waist circumference and waisthip ratio: Report of a WHO expert consultation," Tech. Rep., WHO, Geneva, Switzerland, 2011.

[10] A. T. Hirsch, M. H. Criqui, D. Treat-Jacobson et al., "Peripheral arterial disease detection, awareness, and treatment in primary care," The Journal of the American Medical Association, vol. 286, no. 11, pp. 1317-1324, 2001.

[11] A. D. Blann, F. M. Belgore, C. N. McCollum, S. Silverman, P. L. Lip, and G. Y. H. Lip, "Vascular endothelial growth factor and its receptor, Flt-I, in the plasma of patients with coronary or peripheral atherosclerosis, or type II diabetes," Clinical Science, vol. 102, no. 2, pp. 187-194, 2002.

[12] A. J. Makin, N. A. Y. Chung, S. H. Silverman, and G. Y. H. Lip, "Vascular endothelial growth factor and tissue factor in patients with established peripheral artery disease: A link between angiogenesis and thrombogenesis?" Clinical Science, vol. 104, no. 4, pp. 397-404, 2003.

[13] C. M. Findley, R. G. Mitchell, B. D. Duscha, B. H. Annex, and C. D. Kontos, "Plasma levels of soluble Tie2 and vascular endothelial growth factor distinguish critical limb ischemia from intermittent claudication in patients with peripheral arterial disease," Journal of the American College of Cardiology, vol. 52, no. 5, pp. 387-393, 2008.

[14] K. Yeboah, P. Puplampu, J. Ainuson, J. Akpalu, B. Gyan, and A. G. B. Amoah, "Peripheral artery disease and exertional leg symptoms in diabetes patients in Ghana," BMC Cardiovascular Disorders, vol. 16, no. 1, article 68, 2016.

[15] P. Vempati, A. S. Popel, and F. Mac Gabhann, "Extracellular regulation of VEGF: Isoforms, proteolysis, and vascular patterning," Cytokine \& Growth Factor Reviews, vol. 25, no. 1, pp. 1-19, 2014.

[16] R. Kikuchi, K. Nakamura, S. MacLauchlan et al., "An antiangiogenic isoform of VEGF-A contributes to impaired vascularization in peripheral artery disease," Nature Medicine, vol. 20, no. 12, pp. 1464-1471, 2014.

[17] H. S. Lim, A. D. Blann, A. Y. Chong, B. Freestone, and G. Y. Lip, "Plasma vascular endothelial growth factor, angiopoietin-1, and angiopoietin-2 in diabetes: implications for cardiovascular risk and effects of multifactorial intervention," Diabetes Care, vol. 27, no. 12, pp. 2918-2924, 2004.

[18] S. Nomura, A. Shouzu, S. Omoto et al., "Effects of eicosapentaenoic acid on endothelial cell-derived microparticles, angiopoietins and adiponectin in patients with type 2 diabetes," Journal of Atherosclerosis and Thrombosis, vol. 16, no. 2, pp. 8390, 2009.

[19] S. Anuradha, V. Mohan, K. Gokulakrishnan, and M. Dixit, "Angiopoietin-2 levels in glucose intolerance, hypertension, and metabolic syndrome in Asian Indians (Chennai Urban Rural Epidemiology Study-74)," Metabolism - Clinical and Experimental, vol. 59, no. 6, pp. 774-779, 2010.

[20] S. K. Nadar, A. Blann, D. G. Beevers, and G. Y. H. Lip, "Abnormal angiopoietins 1\&2, angiopoietin receptor Tie-2 and vascular endothelial growth factor levels in hypertension: Relationship to target organ damage [a sub-study of the AngloScandinavian Cardiac Outcomes Trial (ASCOT)]," Journal of Internal Medicine, vol. 258, no. 4, pp. 336-343, 2005.

[21] Y. Reiss, A. Scholz, and K. H. Plate, "The angiopoietin-tie system: common signaling pathways for angiogenesis, cancer, and inflammation," in Endothelial Signaling in Development and Disease, pp. 313-328, Springer, 2015.

[22] B. Hoier, M. Walker, M. Passos et al., "Angiogenic response to passive movement and active exercise in individuals with peripheral arterial disease," Journal of Applied Physiology, vol. 115, no. 12, pp. 1777-1787, 2013.

[23] C. D. Askew, S. Green, P. J. Walker et al., "Skeletal muscle phenotype is associated with exercise tolerance in patients with peripheral arterial disease," Journal of Vascular Surgery, vol. 41, no. 5, pp. 802-807, 2005.

[24] J. L. Robbins, W. Schuyler Jones, B. D. Duscha et al., "Relationship between leg muscle capillary density and peak hyperemic blood flow with endurance capacity in peripheral artery disease," Journal of Applied Physiology, vol. 111, no. 1, pp. 81-86, 2011.

[25] A. V. Benest, A. H. Salmon, W. Wang et al., "VEGF and angiopoietin-1 stimulate different angiogenic phenotypes that combine to enhance functional neovascularization in adult tissue," Microcirculation, vol. 13, no. 6, pp. 423-437, 2006.

[26] D. Qin, T. Trenkwalder, S. Lee et al., "Early vessel destabilization mediated by angiopoietin-2 and subsequent vessel maturation via angiopoietin-1 induce functional neovasculature after ischemia," PLoS ONE, vol. 8, no. 4, article e61831, 2013.

[27] P. C. Bennett, P. S. Gill, S. Silverman, A. D. Blann, B. Balakrishnan, and G. Y. H. Lip, "Ethnic/racial differences in circulating markers of angiogenesis and their association with cardiovascular risk factors and cardiovascular disease," International Journal of Cardiology, vol. 167, no. 4, pp. 1247-1250, 2013.

[28] J. A. Beckman, C. O. Higgins, and M. Gerhard-Herman, "Automated oscillometric determination of the ankle-brachial index provides accuracy necessary for office practice," Hypertension, vol. 47, no. 1, pp. 35-38, 2006.

[29] W. J. Verberk, A. Kollias, and G. S. Stergiou, "Automated oscillometric determination of the ankle-brachial index: A systematic review and meta-analysis," Hypertension Research, vol. 35, no. 9, pp. 883-891, 2012. 


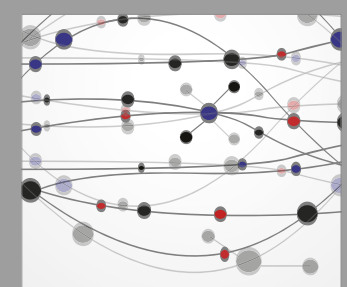

The Scientific World Journal
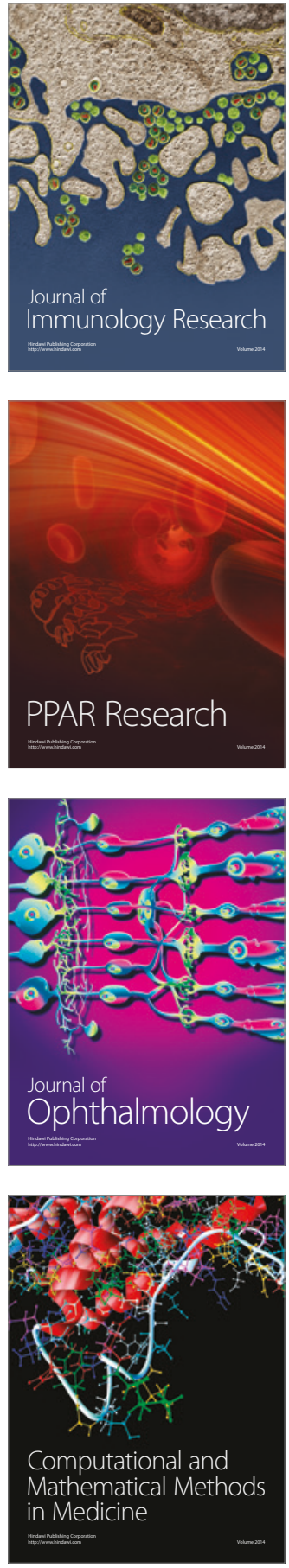

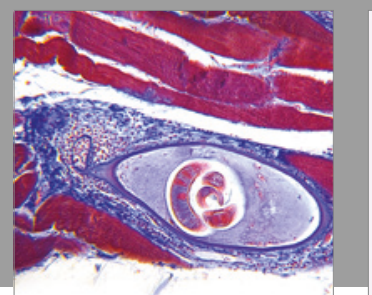

Gastroenterology Research and Practice
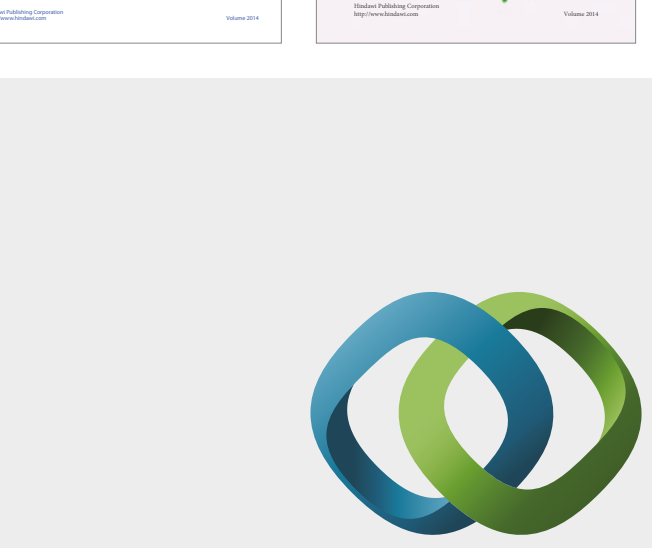

\section{Hindawi}

Submit your manuscripts at

https://www.hindawi.com
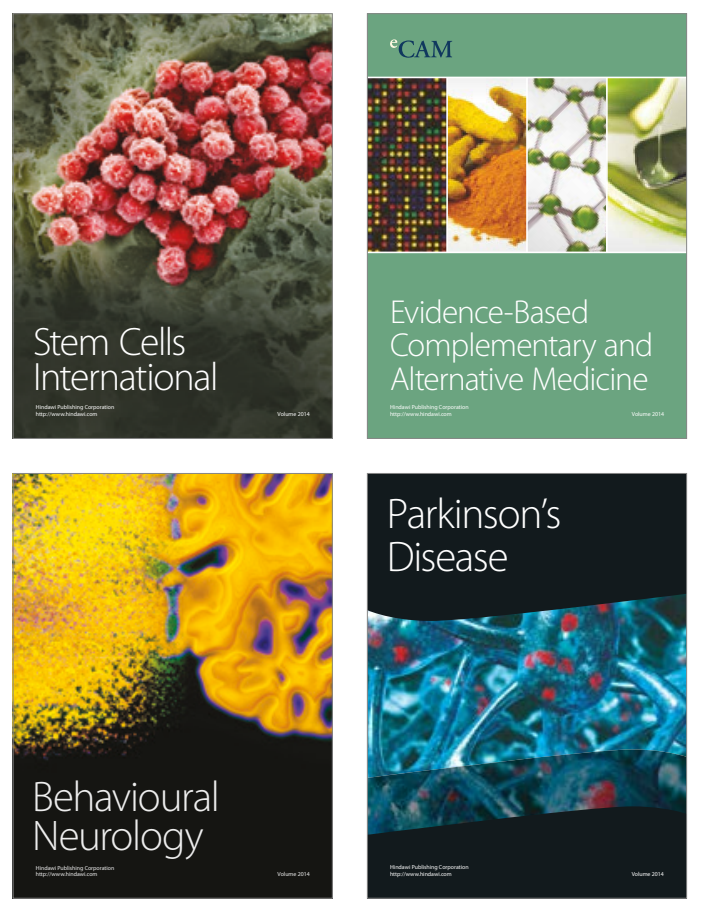
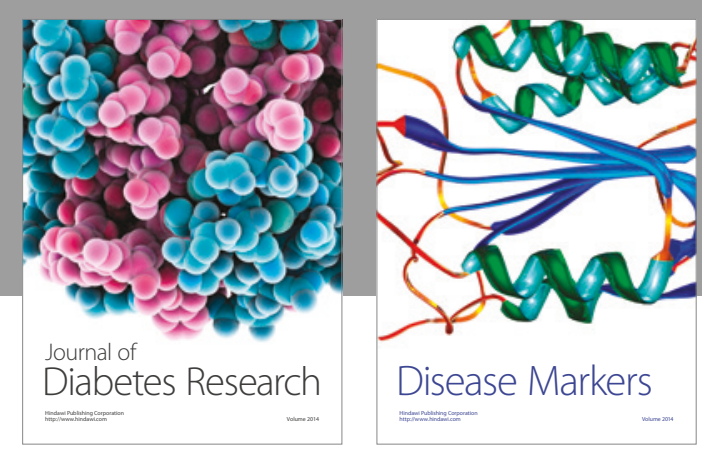

Disease Markers
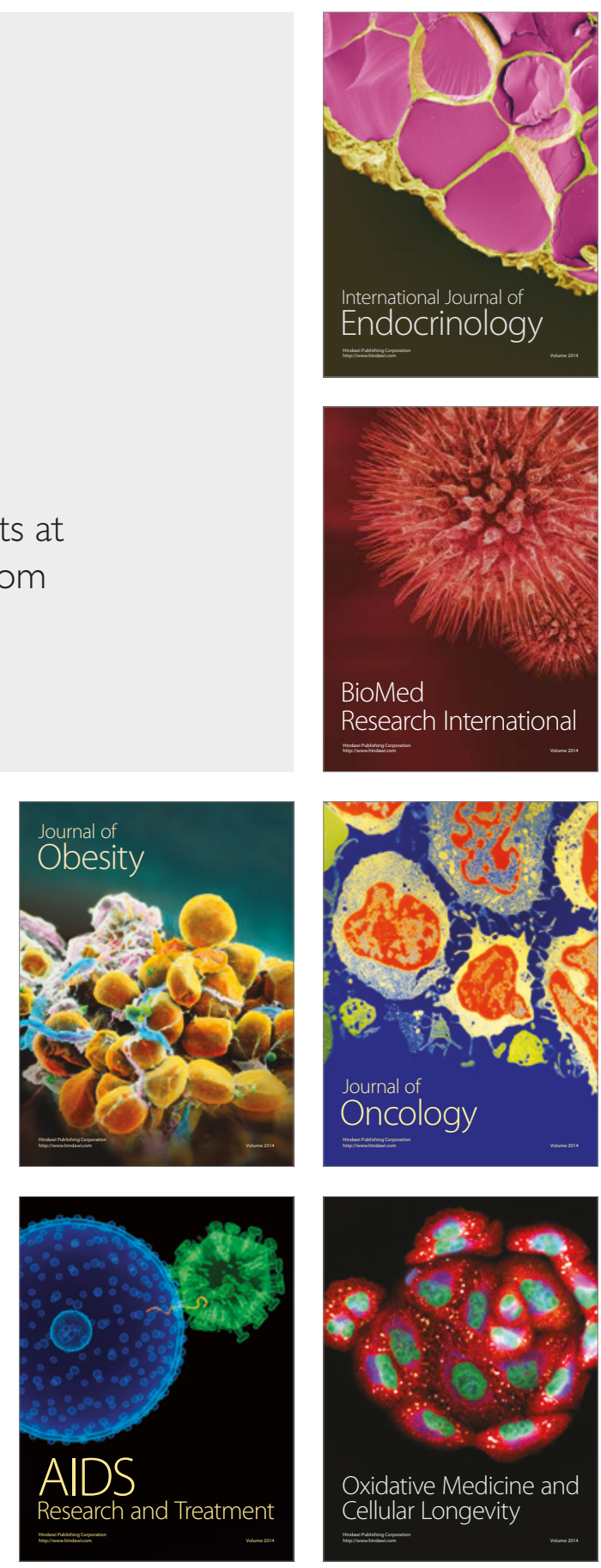